\title{
Provider and Staff Feedback on Screening for Social and Behavioral Determinants of Health for Pediatric Patients
}

\author{
Elena Byboff, MD, MSc, Arvin Garg, MD, MPH, Michelle Pellicer, MPH, \\ Yareliz Diaz, BA, Grace H. Yoon, BA, Martin P. Charns, DBA, and \\ Mari-Lynn Drainoni, PhD
}

Introduction: Screening and referral for Social and Behavioral Determinants of Health (SDOH) are increasingly recommended in clinical guidelines and consensus statements. It is important to understand barriers and facilitators to implementation of standardized SDOH screening and referral practices, as well as the scope of current existing SDOH screening.

Methods: We conducted a mixed-methods study to understand the current state of SDOH screening and to assess the barriers and facilitators to implementing a standardized SDOH screening and referral practice in Boston community health centers (CHCs) for pediatric patients. We requested all SDOH screening documents from 15 Boston CHCs and conducted provider and staff focus groups at intervention sites of an SDOH implementation pilot in Boston.

Results: All CHCs screened in some form for SDOH, but there was no agreement on which domains to screen. Participating CHCs screened for a mean of 8 SDOH domains (range, 5 to 16). Overall, 16 SDOH domains emerged. From the focus groups, 5 themes emerged: 1) provider perspectives, 2) work flow, 3) prior experience, 4) site resources and staffing, and 5) sustainability. There was little agreement among participants within each theme, as all were seen as barriers and facilitators depending on the respondent.

Discussion: This study highlights the various SDOH screening methods currently used in Boston CHCs, and the need for workflow and process individualization of SDOH screening and referral. Providers and clinical staff should be part of the discussion when implementing SDOH screening and referral procedures to ensure appropriate work flow, staff buy-in, and to maximize resources available. ( $\mathrm{J}$ Am Board Fam Med 2019;32:297-306.)

Keywords: Boston, Community Health Centers, Community Medicine, Focus Groups, Healthcare Disparities, Primary Health Care, Public Health, Qualitative Research, Social Determinants of Health, Workflow

There is increasing awareness of the role Social and Behavioral Determinants of Health (SDOH) play in health outcomes and barriers to health equity. The World Health Organization defines SDOH as the conditions in which people are born, grow, live, work and age. ${ }^{1}$ Public health practitioners have long

This article was externally peer reviewed.

Submitted 17 September 2018; revised 10 December 2018; accepted 12 December 2018.

From the Department of Medicine, Tufts Medical Center; Institute for Clinical Research and Health Policy Studies, Tufts CTSI, Boston, MA (EB); Department of Medicine, Tufts University School of Medicine, Boston (EB); Department of Pediatrics, Boston University School of Medicine, Boston Medical Center, Boston (AG, MP); Department of Health Law Policy \& Management, Boston University School of Public Health, Boston (YD, GHY, purported the importance of addressing these social and environmental factors' impact on health ${ }^{2}$; however the implementation of SDOH screening and

MPC, M-LD); VA Center for Healthcare Organization and Implementation Research, Department of Veterans Affairs, Boston, MA (MPC); Section of Infectious Diseases, Evans Center for Implementation and Improvement Sciences, Department of Medicine, Boston University School of Medicine, Boston (M-LD).

Funding: This research was funded by the Eunice Kennedy Shriver National Institute of Child Health and Human Development (NICHD) Grant R01MD007793. Funded by the National Institutes of Health (NIH) and by the Jonathan

M. Tisch College of Civic Life at Tufts University.

Conflict of interest: none declared.

Corresponding author: Elena Byhoff, MD, MSc, Tufts Medical Center, Department of Medicine, 800 Washington Street, Boston, MA 02111 (E-mail: ebyhoff@tuftsmedicalcenter.org). 
referral interventions in a clinical environment is a relatively new phenomenon. Efficacy trials found that SDOH screening and referral interventions increase physician awareness of SDOH prevalence ${ }^{3,4}$ while improving referrals in the targeted population. ${ }^{5}$ With nearly $70 \%$ of variation in health outcomes driven by $\mathrm{SDOH}$, there is great potential that $\mathrm{SDOH}$ screening and referral interventions can improve health at the individual and population levels. ${ }^{6,7}$ Recently, the Centers for Medicare and Medicaid began to incentivize innovation in screening for and addressing $\mathrm{SDOH}$, further increasing its implementation and adoption in medical practices. ${ }^{8}$

The Institute of Medicine's report, Crossing the Quality Chasm $^{9}$, however, described the widespread inability of medicine to implement practice innovations with proven efficacy. It takes an average of 17 years to incorporate advances from clinical research into routine practice. ${ }^{10}$ Screening and referral for SDOH is in the early stages of practice implementation and evaluation. ${ }^{1-13}$ There is a paucity of empirical evidence on implementation of best practices. Evidence to date suggests that providers understand the value of SDOH screening, but find that it is time intensive and poses ethical and logistic challenges, which hinder the implementation of SDOH interventions into practice. $^{3,14-17}$ Finally, it is unclear which specific SDOH domains are being targeted by practices for screening and referral, as there is variation among screening tools. ${ }^{5,18-21}$

Establishing appropriate screening and referral processes for clinical staff is imperative to achieve routine $\mathrm{SDOH}$ screening and referral. ${ }^{1,8,22-25}$ To address this implementation gap regarding integrating SDOH screening and referral interventions, we performed a mixed-methods study in Boston community health centers (CHCs). The qualitative component is an implementation evaluation of the WE CARE screening and referral pilot, described elsewhere. $^{5}$ Our aims were to 1) characterize any current $\mathrm{SDOH}$ screening and referral practices across 15 Boston CHCs, and 2) understand facilitators and barriers in the process of screening and referral implementation of a specific SDOH screening tool in a subset of these CHCs.

\section{Methods}

To address aim 1, we requested and analyzed the SDOH screening forms from 15 Boston CHCs. To address aim 2, we conducted interviews at 3 participating study sites on implementation experiences with the WE CARE SDOH screening tool. ${ }^{5} \mathrm{We}$ interviewed CHC providers and staff, and coded those interviews for facilitators and barriers.

This study was conducted in partnership with the Center for Community Health Education Research and Service (CCHERS), a community organization representing $15 \mathrm{CHCs}$ in Boston. ${ }^{26}$ The study was approved by the Boston University Medical Center and Tufts Medical Center Institutional Review Board.

\section{Current Screening Practices in Boston CHCs SDOH Screening Data Collection and Domain Development}

From September 2017 to January 2018, the study team requested copies of any forms used to screen for SDOH at all 15 CCHERS member CHCs. We also requested data on screening form implementation 1) when during the visit patients were screened, 2) who completes the screening form, 3) types of patient visits (new patient, return patient, acute visit, other), and 4) any specific patient population being targeted for screening (adult, pediatric, prenatal, other).

\section{Coding and Content Analysis}

Content analysis was used to code the submitted screening forms into cohesive domains and dimensions. Domains of SDOH included both social and behavioral determinants of health, and were based on prior work and published national initiatives and were revised by the research team based on preliminary coding of a subset of submitted screening forms. ${ }^{21} \mathrm{We}$ included any references to social or behavioral determinants as SDOH. Codes were separated into "domains" representing larger categories of screening questions that were comprised of "dimensions"-more specific screening questions. Content analysis is a research method that can be used to code and quantify data from documents that were not developed primarily for analytic purposes. ${ }^{27,28}$ Three coders (EB, GY, YD) used content analysis to assign SDOH domains to each screening form. All forms were reviewed by a minimum of 2 coders. There was $\geq 95 \%$ concordance between coders, and all differences between coders were adjudicated by all members of the coding team. A priori, any forms submitted with a medical intent rather than a screening intent were 
excluded from coding. Examples of such excluded documents were screening documents for Autism Spectrum Disorder or prenatal medical history. All coding documents that had a single screening intent were assigned one code, even if they consisted of multiple questions that could have each received codes. For example, the Patient Health Questionnaire (PHQ) 9 was given a single code for "depression."

\section{Facilitators and Barriers to SDOH Screening Implementation \\ SDOH Screening and Referral Implementation}

Before the WE CARE screening and referral implementation, 8 CCHERS member CHCs were identified as having no formalized SDOH screening and referral process in place. ${ }^{5}$ Six agreed to participate in a cluster randomized control trial to implement and examine the effectiveness of a screening and referral protocol for SDOH during well-child visits; 3 pediatric clinics implemented the WE CARE intervention beginning in September 2015 , the other 3 served as control sites. ${ }^{5}$ The WE CARE practice workflow consisted of the following: 1) distribution of a paper WE CARE screener by medical assistants; 2) medical assistants entered responses into the electronic medical record; 3) providers or practice staff printed resource information sheets for parents; and 4) if interested, parents would be referred to a patient navigator for additional assistance.

\section{Qualitative Instrument Development and Data Collection}

We visited the 3 intervention sites to gather practitioner and staff perspectives on the context, implementation, and delivery of WE CARE during the pilot implementation phase, just before it became the standard of care. The Promoting Action on Research Implementation (PARIHS) framework was used to develop and design our data collection instrument. PARIHS is a conceptual framework that helps explain why implementing evidence into practice may or may not be successful by including the relationship between the evidence, the implementation context (in this case our clinic) and facilitation. ${ }^{29,30}$

The semistructured interview guide focused on understanding participants' knowledge of current SDOH screening practices and how the WE CARE screening and referral process compared with the previous standard of care within their
CHC. We asked contextual questions about how the adoption of new practices within their setting usually worked. We also explored factors that were impacted by implementation, including staff roles and responsibilities, logistical challenges, patient challenges, and the processes by which the facilitation occurred. Finally, we asked participants about the implementation process by the study team. In total, 6 focus groups were conducted between July and August 2015 at intervention sites from the original study. Each site had 2 focus groups-1 with pediatric clinicians (physicians and nurses) and 1 with medical assistants. All sessions were conducted by a single facilitator, recorded, and professionally transcribed; the software program NVivo (QSR International Pty Ltd. Version 11, 2015) was used for transcript coding and data management. ${ }^{31}$

\section{Qualitative Analysis}

To conduct the qualitative data analysis, we used grounded theory and constant comparative methods. ${ }^{32,33}$ Two team members inductively analyzed the transcripts line by line to create codes to characterize comments and passages. The transcripts were then re-evaluated to group codes into concepts. The whole research team discussed the findings and developed a cohesive coding scheme, which was iteratively refined with review and coding of subsequent transcripts. After consensus was established, each of the remaining transcripts were coded, followed by discussion of coding discrepancies and arrival at final coding decisions. The authors completed final analyses, identifying overall themes and concepts, and selecting representative quotations. We mapped the emergent themes as either facilitators or barriers to the implementation of WE CARE.

\section{Results}

\section{CHC Participant Characteristics}

We received social and behavioral screening documents from $87 \%(n=13)$ of all CCHERS affiliated Boston CHCs, including the 3 CHCs from the $\mathrm{SDOH}$ screening and referral pilot who also participated in the study focus groups. Patient demographic data and clinic characteristics were available for 11 of the 13 participating CHCs (Table 1). The 3 sites participating in the focus groups were similar to the other participant sites, with a slightly higher percentage of pediatric visits $(30 \%$; range, 


\begin{tabular}{lcc}
\hline & $\begin{array}{c}\text { All Participating Health Centers, } \\
\text { Mean (Range) }\end{array}$ & $\begin{array}{c}3 \text { Implementation Sites, Mean } \\
\text { (Range) }\end{array}$ \\
\hline Total annual patient visits & $23,271(9,553-70,603)$ & $17,726(12,410-21,518)$ \\
$\%$ of patients $<18$ & $25 \%(16-32)$ & $30 \%(27-32)$ \\
Patients of racial and/or ethnic minority & $75 \%(21-98)$ & $91 \%(87-95)$ \\
Patients at or below 200\% of FPL & $85 \%(47-100)$ & $96 \%(93-100)$ \\
Uninsured & $18 \%(6-31)$ & $13 \%(11-17)$ \\
Medicaid/CHIP 2 & $49 \%(35-61)$ & $51 \%(42-56)$ \\
Dually eligible (Medicare and Medicaid) & $3 \%(2-7)$ & $2 \%(2-3)$ \\
Medical visits & $81 \%(51-98)$ & $83(82-85)$ \\
Dental visits & $26 \%(5-45)$ & $21(17-25)$ \\
Mental health visits & $7 \%(2-11)$ & $6(2-10)$ \\
\hline
\end{tabular}

Data taken from publicly available Health Resources and Services Administration uniform data system or reported by individual health center for 11 of 13 participating health centers-2 health centers were hospital affiliated and did not release patient or visit level demographic data.

CHIP, Children's Health Insurance Program; FPL, Federal Poverty Level.

$27 \%$ to $32 \%$ ), more racial and ethnic minority patients, and a higher percentage of patients living below 200\% of the Federal Poverty Level (FPL).

\section{Boston SDOH Screening Practices}

We identified 16 domains and 78 dimensions of SDOH from the screening documents submitted by participant $\mathrm{CHCs}$ (Table 2). Participating CHCs screened for a mean of $8 \mathrm{SDOH}$ domains (range, 5 to 16). Figure 1 demonstrates the differences in the number of domains screened for in adults compared with pediatric patients in participating CHCs. Three CHCs (23\%) reported only screening for $\mathrm{SDOH}$ in pediatric populations, with 1 (8\%) participating CHC screening only for adults. Of the $9(69 \%)$ CHCs that reported screening both children and adults during clinical visits, all but 1 screened for the same total number of domains. Of the 3 intervention sites for the WE CARE implementation and qualitative review, 2 sites reported only screening for pediatric visits, while the third screened for both adults and children (Figure 1). Figure 2 notes that not all domains were equally screened for in adult compared with pediatric visits. Mental health, health behaviors, and employment were the only domains included in the same number of pediatric and adult screening forms, while the remaining 12 domains were screened for more frequently in the adult setting. Housing was the only domain screened for in all participating CHCs.

\section{WE CARE Implementation Site Focus Group Overview and Themes}

A total of 26 individuals from the $3 \mathrm{WE}$ CARE pilot CHCs participated in 6 focus groups, including 9 physicians, 3 nurses, and 14 medical assistants. The focus groups ranged in size from 3 to 9 participants each. Five themes that emerged were seen as both facilitators and barriers during WE CARE implementation: 1) provider perspective on the intervention, 2) work flow, 3) prior experience, 4) site resources and staffing and 5) sustainability. There was little agreement among focus group participants within each theme, as all were seen as barriers and facilitators depending on the respondent.

\section{Provider Perspective on the Intervention}

Most providers and staff reported positive experiences with both the screening tool and the resource information sheets. Some focus group participants found the screener helpful, particularly that it was available in multiple languages, while others were concerned that the questions were asked too often of parents coming in for visits. One participant stated, "If I were a parent, I would say it is stupid. Why are you bothering me? I filled this out before. . I would not even look at it. I'd throw it away." Another felt that the screening tool allowed for important conversations to take place between the patient and provider that might not happen otherwise. "Sometimes they come in and they do not ask. 
Table 2. Empirically identified SDOH Domains from Health Center Screening Documents

\begin{tabular}{|c|c|}
\hline SDOH Domains* & Dimensions \\
\hline Culture & $\begin{array}{l}\text { Religion/spiritual beliefs } \\
\text { Family culture }\end{array}$ \\
\hline Demographics & $\begin{array}{l}\text { Gender/sexual orientation } \\
\text { Place of birth } \\
\text { Race/ethnicity } \\
\text { Refugee status } \\
\text { Justice involvement }\end{array}$ \\
\hline Economic indicators & $\begin{array}{l}\text { Income } \\
\text { Assets } \\
\text { Assistance programs } \\
\text { Indebtedness }\end{array}$ \\
\hline Education & $\begin{array}{l}\text { Educational attainment } \\
\text { Basic literacy } \\
\text { Health literacy } \\
\text { Numeracy }\end{array}$ \\
\hline Employment & $\begin{array}{l}\text { Unemployed } \\
\text { Migrant/seasonal } \\
\text { Day laborers } \\
\text { Disability status } \\
\text { Retirement status } \\
\text { Student } \\
\text { Job assistance }\end{array}$ \\
\hline Family & $\begin{array}{l}\text { Marital status } \\
\text { Dependents } \\
\text { Living arrangements }\end{array}$ \\
\hline Functional status & $\begin{array}{l}\text { ADLs } \\
\text { IADLs } \\
\text { Frailty }\end{array}$ \\
\hline Health behaviors & $\begin{array}{l}\text { Alcohol } \\
\text { Drug use } \\
\text { Tobacco } \\
\text { Secondhand smoking } \\
\text { Physical activity } \\
\text { Sexual activity } \\
\text { Safety } \\
\text { Diet }\end{array}$ \\
\hline Healthcare access & $\begin{array}{l}\text { Insurance status } \\
\text { Healthcare affordability } \\
\text { Source of usual care }\end{array}$ \\
\hline Housing & $\begin{array}{l}\text { Homelessness } \\
\text { Housing safety } \\
\text { Housing quality } \\
\text { Housing insecurity }\end{array}$ \\
\hline Language & $\begin{array}{l}\text { Primary language } \\
\text { English proficiency } \\
\text { Interpreter/translator needed } \\
\text { Other language proficiency }\end{array}$ \\
\hline
\end{tabular}

Continued
Table 2. Continued

\begin{tabular}{|c|c|}
\hline SDOH Domains* & Dimensions \\
\hline Material hardship & $\begin{array}{l}\text { Food insecurity } \\
\text { Utilities } \\
\text { Transportation } \\
\text { Medication affordability } \\
\text { Access to technology } \\
\text { Child care } \\
\text { Clothing } \\
\text { Legal services }\end{array}$ \\
\hline Mental health & $\begin{array}{l}\text { Depression } \\
\text { Anxiety } \\
\text { PTSD } \\
\text { ADD/ADHD } \\
\text { Suicide/Self-harm } \\
\text { Stress } \\
\text { Sleep }\end{array}$ \\
\hline Social support & $\begin{array}{l}\text { Community activities } \\
\text { Safe environment } \\
\text { Public spaces } \\
\text { Racism } \\
\text { Discrimination } \\
\text { Trust } \\
\text { School culture } \\
\text { Social isolation }\end{array}$ \\
\hline Trauma/violence & $\begin{array}{l}\text { IPV } \\
\text { Trauma } \\
\text { Physical abuse } \\
\text { Sexual abuse } \\
\text { Mental abuse }\end{array}$ \\
\hline Veteran status & $\begin{array}{l}\text { Military trauma history } \\
\text { Combat veteran } \\
\text { Active military }\end{array}$ \\
\hline
\end{tabular}

ADD/ADHD, Attention deficit disorder/hyperactivity disorder; ADL, activity of daily living; IADL, independent activities of daily living; IPV, interpersonal violence; PTSD, Post-traumatic stress disorder; SDOH, Social and Behavioral Determinants of Health.

*All core domains had a "General" subdomain code if screening questions did not pertain to one of the listed subdomains on the right column.

Sixteen domains.

Seventy-eight dimensions.

Even if they are suffering, they do not ask. It is when we talk to them, we ask them a question, that is when they start to open up. With this survey right here, it is very helpful."

\section{Work Flow}

Focus group participants diverged in their perception of level of difficulty and additional time to add WE CARE screening into clinical work flow. Sev- 
Figure 1. Number of domains screened for by each participating community health center (CHC) for pediatric visits compared with any visit.

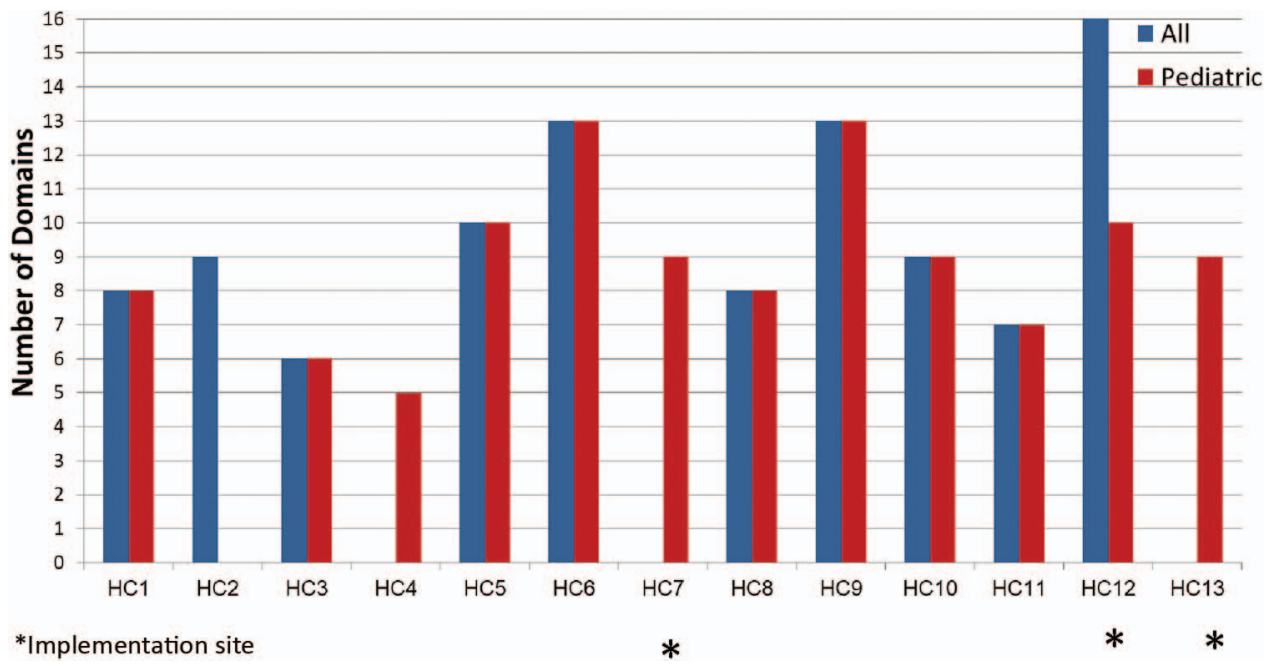

eral providers stated it took no additional time and felt that ". . . having the medical assistants do more screening than they would normally be doing, I think is a good thing." Others felt that additional screening was too time consuming for a busy clinic, stating, "Our [Medical Assistants] will not have time to sit down with each person and do the stuff. ... I do not think there's any chance, especially if it is a busy clinic." Participants also described confusion regarding the referral processes. Some felt it was not clear when a referral was needed and to whom on the care team a patient should be referred.

Interviewee 1: “. . you call the case manager on the day that [the patient navigator] is here, which is Thursdays"

Interviewer: "The case manager or the navigator?"

Interviewee 2: "[we call] the case manager. . Monday through Friday. The case managers are aware of when our navigator is here and if the patients are [the right age for the study]. . . But I've only had 1

Figure 2. Number of community health centers (CHCs) screening for each social \& behavioral domain by adult vs. pediatric visits.

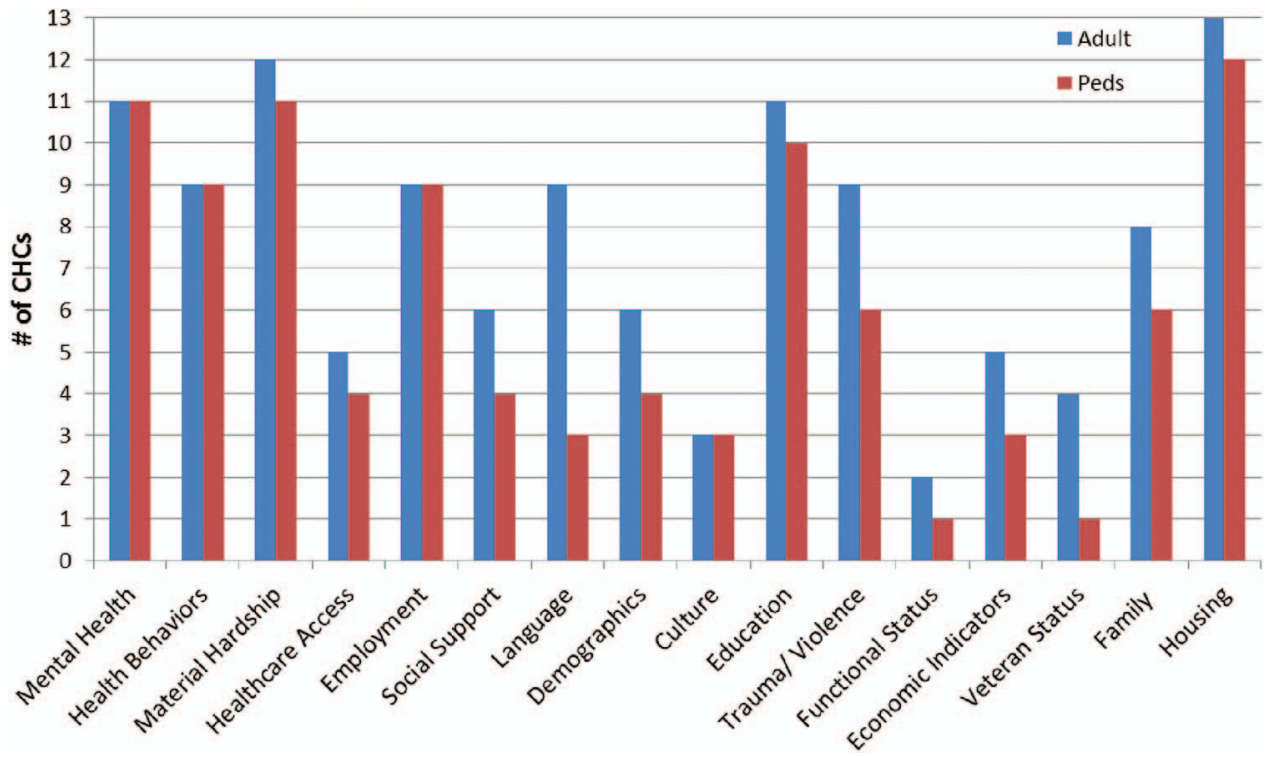


patient where [the patient navigator] was here on the day that I called."

\section{Prior Experience}

Given the demographics and socioeconomic status of the population served by the WE CARE intervention $\mathrm{CHCs}$, many participants had prior experience with existing psychosocial screening and assisting patients with social services. The participants felt that this survey formalized some of the work that was already being done ad hoc in the clinic, and expressed agreement these experiences with both prior psychosocial screening as well as more general research were facilitators in the implementation of $\mathrm{SDOH}$ screening and referrals. All participating $\mathrm{CHCs}$ are also affiliated with a major academic medical center in Boston. As such, the providers and staff reported familiarity with research processes, contributing to positive provider feedback on research protocols. Providers and support staff felt that participating in research was beneficial to patients. One participant stated: “. . . when we participate in pilots like this? I think we like it. We find it helpful. We often incorporate it ongoing."

\section{Site Resources and Staffing}

Despite some concerns about work flow, participants agreed that addition of a patient navigator was positive, despite the limited availability of the navigator. Some sites reported minimal interaction with the navigator, while others found the navigators helpful to identify previously underutilized community resources. Many participants reported that they felt their site had resources available to address identified needs using the screening tool, which was perceived as a facilitating factor for SDOH screening. "We have a very robust social service. . . When patients enroll here for care they do know that we have all these support services that they can access." Another participant stated, "Things come up organically and people already know that this is a place where they can come to. Feel comfortable bringing up issues." Others cited as a barrier a specific lack of resources due to system level issues and this was not something that could be overcome by implementing a screening and referral program. "... when we [send] people to the advocates... they come back and say either there was not really anything they could do. I make too much money to qualify for food stamps. The housing list is a year long."

\section{Sustainability of the Intervention}

Focus group participants reported that they felt the ease of screening and printing out referral resource guides from the electronic medical records was a sustainable approach. Several reported that electronic access to resources via the study implementation improved their ability to offer resources to all patients, noting that before the study, there was a resource binder that was hard to find and out of date. Some participants questioned the efficacy of the intervention before being willing to implement it practice wide. There were differing levels of enthusiasm among providers for sustaining the intervention, which may reflect variations in knowledge of the study protocol and purpose. During several focus groups, when asked what participants knew about the WE CARE study, several responded "not much" or "nothing" making it difficult to comment on sustainability of the intervention.

\section{Discussion}

With increasing focus on SDOH screening and referral interventions, it is critically important to establish implementation best practices. Concerns have been raised about the time and ability of health care sites to both screen for SDOH in an empathic and ethically sound way, using patientcentered, strengths-based approaches with access to referrals and resources, while not increasing administrative tasks for already overburdened primary care providers. ${ }^{17,34}$ The focus groups were part of a larger effectiveness study of the WE CARE pilot that showed positive social outcomes for mothers screened and referred for SDOH during pediatric visits. ${ }^{5} \mathrm{We}$ found no agreed on barriers and facilitators for what could substantially improve the implementation of screening and referral for SDOH. Yet, we found broad uptake of SDOH screening and referral practices across Boston CHCs.

The lack of agreement on facilitators and barriers to implementation points to the absence of specific structural barriers, highlighting the existence of individual styles and preferences of providers and staff and contextual factors. Differences in views expressed about the implementation process were largely between sites, rather than differences between providers and clinical staff. This important finding suggests that tailoring processes 
and including staff/provider buy in for implementation may overcome identified time/work flow/ knowledge barriers. In our study, the implementation protocol was conceived and designed by outside researchers without specific knowledge of the culture at each participating site. In addition, each site used a different electronic medical record. To maintain fidelity to study protocol, all sites completed SDOH screening on paper, which may have limited integration and ease of implementation at the study sites. It will be important to consider involvement of clinic leadership and frontline staff to best incorporate clinical practices and ensure all providers and staff are knowledgeable and invested in study protocol and expectations.

Increased reporting of screening and referral for $\mathrm{SDOH}$ could be explained by several concurrent factors. First, there has been increased attention in the medical community to the importance of $\mathrm{SDOH}$ in the peer-reviewed literature and the lay press. ${ }^{11,35,36}$ Since the implementation of WE CARE screening began in 2015, the American Academy of Pediatrics formalized guidelines to screen for poverty-related conditions at well-child visits. ${ }^{37}$ There have also been significant policy and payment shifts toward identifying and addressing $\mathrm{SDOH}$ in health care, including the Accountable Health Communities model and the Massachusetts Medicaid demonstration project. ${ }^{38,39}$ In particular, we have noted that while pediatric patients make up the minority of those seeking care in our sample (Table 1), participating CHCs are largely including the same number of domains in both adult and pediatric visits (Figure 1). This signals that CHCs are screening comprehensively across patients of all ages. Three sites reported screening for SDOH in pediatric visits only. Along with broader movement in the medical field toward increasing formalized SDOH screening and referral, there may have been diffusion from our cohort of CHCs to prioritize pediatric screening.

Our findings highlight a range of $\mathrm{SDOH}$ domains and dimensions that are being screened for, without clear consensus for a single screening tool or agreement on particular screening questions. The most frequently screened domains included housing $(\mathrm{n}=$ 13), material hardship $(\mathrm{n}=12)$, and mental health $(\mathrm{n}=11)$, which are in alignment with national recommendations. ${ }^{19,21}$ Despite this broad agreement between Boston $\mathrm{CHCs}$ and national policy makers, few validated screening questions exist and therefore SDOH domain alignment does not necessarily correlate to comparable screening items. The identified diversity of SDOH domains in our data may signal differences in clinical sites' priorities for screening and referral. This may be due to onsite resource availability, specialized staff, local partnerships or other unique aspects of practice. As national guidelines and professional organizations recommend adoption of SDOH screening and referral in routine clinical practice, it will be important to enable tailoring for clinical sites to implement $\mathrm{SDOH}$ screening and referral practices to their strengths or perceived patient needs.

Limitations of this study include results from 1 city that might not be generalizable, yet other studies have shown similar results with regard to general acceptance of SDOH screening and referral. ${ }^{40}$ The WE CARE implementation and evaluation took place in pediatric clinics only. Processes and workflow in pediatrics might not be generalizable to all primary care settings. The implementation evaluation focused on provider feedback and experiencing, thus limiting our ability to comment on patient perspectives. Our study took place in CHCs, which may not be representative of all primary care environments. CHCs are a reasonable place to evaluate SDOH screening practices based on patient population and a mission to serve socioeconomically disadvantaged populations. CHCs often include colocated mental health or social services relevant to $\mathrm{SDOH}$ screening which may make CHCs unique and more likely to participate in SDOH screening compared with other primary care practices. Our data highlights the conflation by providers and staff of social determinants of health (ie, material hardship, food insecurity), behavioral determinants (ie, alcohol use, tobacco use) and mental health screening (ie, depression, stress, anxiety). While each has a distinct impact on physical health, we have included all these factors as "social and behavioral" determinants for the purposes of our analysis. We requested all $\mathrm{SDOH}$ screening materials used by each clinic, which was up to interpretation by the individual providing the documents and therefore might not have received everything that each site was using with respect to SDOH screening and referral. Finally, qualitative studies are hypothesis generating, and our sample may have respondent bias, but variation in responses show good representation of perspectives. 
Despite not identifying successes or hurdles to SDOH screening and referral implementation, we found increased SDOH screening generally. To develop best practices in SDOH screening and referral, future work should incorporate feedback from the clinical team to improve the implementation plan, further educate site staff on study purpose and protocol, improve integration of the patient navigator into care teams, and report study outcomes to motivate further participation by providers and clinical staff. Engaging providers and patients in prioritizing SDOH screening domains to maximally benefit the patient population may encourage more provider agreement in routine SDOH screening protocols. Ongoing opportunities for provider and staff feedback will improve work flow and protocol buy in, while allowing for site specific tailoring to clinic resources and patient needs.

The authors would like to thank Elmer Freeman at the Center for Community Health and Education Services for data collection assistance.

To see this article online, please go to: http://jabfm.org/content/ 32/3/297.full.

\section{References}

1. World Health Organization. A conceptual framework for action on the social determinants of health. The discussion paper series on social determinants of health. 2010. Available from: http://www.who.int/ sdhconference/resources/Conceptualframeworkfor actiononSDH_eng.pdf.

2. Marmot M, Friel S, Bell R, Houweling TA, Taylor $\mathrm{S}$. Closing the gap in a generation: Health equity through action on the social determinants of health. Lancet 2008;372:1661-9.

3. Tong ST, Liaw WR, Kashiri PL, et al. Clinician experiences with screening for social needs in primary care. The J Am Board Fam Med 2018;31: 351-63.

4. Garg A, Jack B, Zuckerman B. Addressing the social determinants of health within the patient-centered medical home: Lessons from pediatrics. JAMA 2013; 309:2001-2.

5. Garg A, Toy S, Tripodis Y, Silverstein M, Freeman E. Addressing social determinants of health at well child care visits: A cluster RCT. Pediatrics 2015; 135(2):e296-e304.

6. Schroeder SA. We can do better-Improving the health of the American people. N Engl J Med 2007; 357:1221-8.

7. Woolf SH, Braveman P. Where health disparities begin: The role of social and economic determi-
nants-And why current policies may make matters worse. Health Aff (Millwood) 2011;30:1852-9.

8. Alley DE, Asomugha CN, Conway PH, Sanghavi DM. Accountable health communities-Addressing social needs through medicare and medicaid. N Engl J Med 2016;374:8-11.

9. Wolfe A. Institute of Medicine Report: Crossing the quality chasm: A new health care system for the $21 \mathrm{st}$ century. Policy Politics Nurs Pract 2001;2:233-5.

10. Morris ZS, Wooding S, Grant J. The answer is 17 years, what is the question: Understanding time lags in translational research. J Royal Soc Med 2011;104: 510-20.

11. Gold R, Cottrell E, Bunce A, et al. Developing electronic health record (EHR) strategies related to health center patients' social determinants of health. The J Am Board Fam Med 2017;30:428-47.

12. Pinto AD, Glattstein-Young G, Mohamed A, Bloch G, Leung FH, Glazier RH. Building a foundation to reduce health inequities: Routine collection of sociodemographic data in primary care. J Am Board Fam Med 2016;29:348-55.

13. LaForge K, Gold R, Cottrell E, et al. How 6 organizations developed tools and processes for social determinants of health screening in primary care: An overview. J Ambul Care Manage 2018;41:2-14.

14. Mooney K, Moreno C, Chung PJ, Elijah J, Coker TR. Well-child care clinical practice redesign at a community health center: Provider and staff perspectives. J Prim Care Community Health 2014;5: 19-23.

15. Palacio A, Seo D, Medina H, Singh V, Suarez M, Tamariz L. Provider perspectives on the collection of social determinants of health. Popul Health Manag 2018;21:501-8.

16. Garg A, Sheldrick RC, Dworkin PH. The inherent fallibility of validated screening tools for social determinants of health. Acad Pediatr 2018;18:123-4.

17. Garg A, Boynton-Jarrett R, Dworkin PH. Avoiding the unintended consequences of screening for social determinants of health. JAMA 2016;316:813-4.

18. PRAPARE. Research and Data. 2018. Available from: http://www.nachc.org/research-and-data/prapare/.

19. Center for Medicare and Medicaid Innovation. The accountable health communities health-related social needs screening tool. Available from: https://innovation.cms.gov/Files/worksheets/ahcmscreeningtool.pdf.

20. Billioux A, Verlander K, Anthony S, Alley D. Standardized screening for health-related social needs in clinical settings: The Accountable Health Communities screening tool. 2017. Available from: https:// nam.edu/standardized-screening-for-health-relatedsocial-needs-in-clinical-settings-the-accountablehealth-communities-screening-tool/.

21. Giuse NB, Koonce TY, Kusnoor SV, et al. Institute of Medicine Measures of Social and Behavioral De- 
terminants of Health: A feasibility study. Am J Prev Med 2017;52:199-206.

22. Bogard K, McBride Murry V, Alexander C. Perspectives on health equity and social determinants of health. 2017. Available from: https://nam.edu/wpcontent/uploads/2017/12/Perspectives-on-HealthEquity-and-Social-Determinants-of-Health.pdf.

23. Centers for Disease Control and Prevention. Policy resources to support social determinants of health. Social determinants of health. 2017. Available from: https://www.cdc.gov/socialdeterminants/policy/ index.htm.

24. Berman RS, Patel MR, Belamarich PF, Gross RS. Screening for poverty and poverty-related social determinants of health. Pediatr Rev 2018;39:235-46.

25. Daniel H, Bornstein SS, Kane GC, for the H. Addressing social determinants to improve patient care and promote health equity: An American College of Physicians position paper. Ann Intern Med 2018; 168:577-8.

26. Center for Community Health Education Research and Service. About us. 2018. Available from: https:// www.cchers.org/about.

27. Elo S, Kyngäs H. The Qualitative content analysis process. J Adv Nurs 2008;62:107-15.

28. Krippendorff K. Measuring the reliability of qualitative text analysis data. Qual Quant 2004;38:787-800.

29. Harvey G, Kitson A. PARIHS revisited: From heuristic to integrated framework for the successful implementation of knowledge into practice. Implement Sci 2017;11:33.

30. Rycroft-Malone J. The PARIHS framework-A framework for guiding the implementation of evi- dence-based practice. J Nurs Care Qual 2004;19: 297-304.

31. NVIVO. What is NVivo. 2018. Available from: https://www.qsrinternational.com/nvivo/what-is-nvivo.

32. Pope C, Ziebland S, Mays N. Qualitative research in health care. Analysing qualitative data. BMJ 2000; 320:114-6.

33. Glaser BG, Strauss AL. The discovery of grounded theory: Strategies for qualitative theory. Chicago, IL: Aldine Publishing Company; 1967.

34. Desalvo KB, Muntner P. Discordance between physician and patient self-rated health and all-cause mortality. Ochsner J 2011;11:232-40.

35. Byhoff E, Freund KM, Garg A. Accelerating the implementation of social determinants of health interventions in internal medicine. J Gen Intern Med 2018;33:223-5.

36. Khullar D. Is it getting harder to care for poor patients? New York Times. 6/26/2018.

37. Gitterman BA, Flanagan P, Cotton W, et al. Poverty and child health in the United States. Pediatrics 2016;137(4) pii: e20160339.

38. Centers for Medicare and Medicaid Services. Accountable health communities model. Innovation models. 2018. Available from: https://innovation. cms.gov/initiatives/ahcm/.

39. 1115 MassHealth Demonstations ("Waiver"). 2018. Available from: https:/www.mass.gov/service-details/ 1115-masshealth-demonstration-waiver.

40. Adler NE, Glymour MM, Fielding J. Addressing social determinants of health and health inequalities. JAMA 2016;316:1641-2. 